\title{
Improvement on Driving Comfort and Energy Consumption of Electric Vehicle through Throttle Signal Control
}

\author{
Zhang Kun, Joerg D. Weigl \\ National University of Singapore, Faculty of Engineering, Engineering Design and Innovation Centre, Design Centric \\ Program, Block E1A, \#03-03,1Engineerrind Drive2, Singapore 117576, zhang_kun@u.nus.edu
}

\begin{abstract}
An electric vehicle conversion project often faces the problem of a sudden and non-smooth acceleration. From this, we see a great demand in improving the throttle control to have a better human-machine interface and better controllability and safe operation of an electric vehicle. Additional improvement on throttle control also helps to reduce energy consumption during acceleration which prolongs the driving range of the vehicle.

This work presents a practical method to provide a smooth start for an electric vehicle with brushless DC (BLDC) motor using arduino microcontroller. Additional control algorithms between the throttle and motor controller are implemented with the microcontroller. Two sets of algorithm, which can be used at the same time, have been developed in order to reach the goal of smoother acceleration, better controllability, and lower energy consumption. Tests have been carried out on our in-house developed vehicle, Electric Caterham, to validate the effectiveness of different algorithms. We expect a $10 \%$ reduction in energy consumption during acceleration. This paper presents the change in acceleration performance after implementing the proposed feedback control system.
\end{abstract}

Keywords: Throttle control; Microcontroller; Feedback control; Hall Effect sensor

\section{Introduction}

Due to growing concern of energy crisis and global warning, electric vehicle, especially battery electric vehicle (BEV) market has grown rapidly in recent years. [1] One advantage that BEV has over internal combustion engine vehicle (ICEV) is that weight reduction due to simplified power train. [2] However, over simplification of the power train also remove some desirable functionalities, such as smooth acceleration through clutch and gearbox, of conventional power train. In an ICEV, a clutch between the engine and transmission is necessary because the engine usually starts working at a non-zero rpm.
When the clutch on an ICEV engages, the transmission system is accelerated to engine speed from zero rpm. During this process, the torque transmitted to the wheels build up gradually. The presence of gearbox also make throttle control of ICEV easier as the acceleration is divided into several segments, which ensure the drivability. All these functionalities of traditional transmission system need to be compensated by the throttle control and motor control system of BEV. For $\mathrm{BEV}$, these are crucial because a BLDC responds fast to input signal change. [3] A project is ongoing to modify the throttle control system of a $\mathrm{BEV}$ to achieve better driving experience and more 
functionality.

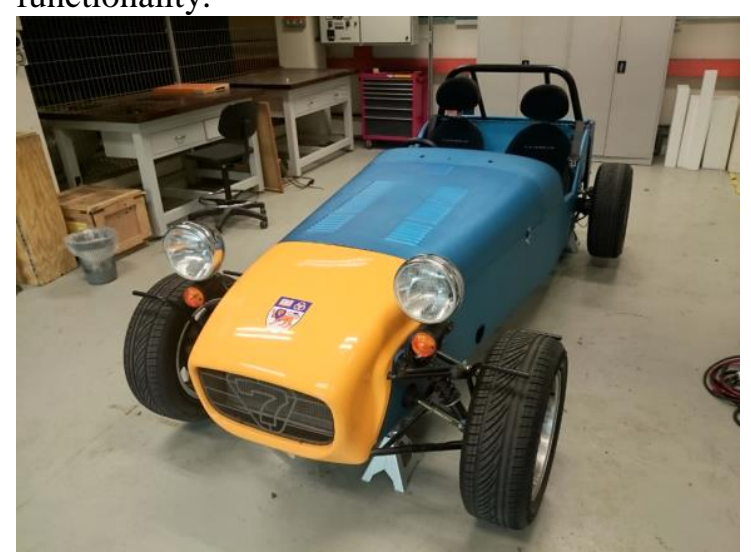

Figure 1: Electric Caterham

The BEV, Electric Caterham (E-Cat), is an electric conversion project ongoing in National University of Singapore (NUS). E-Cat, which is converted from a Classic Caterham 7, is powered by two $5 \mathrm{~kW}$ BLDC motor. Currently, a position sensor is built into the throttle, whose voltage varies as the throttle position changes. The voltage signal is transmitted to both motor controllers through an Arduino Mage 2560 microcontroller. Previously, the microcontroller passes the exact signal from the position sensor the motor controllers. By adding feedback algorithm, which adjusts the throttle signal, to the microcontroller, the desired functionalities may be achieved.

\section{Feedback control system}

Two different sensors has been used to build the feedback control system.

Proposed control system consist of a sensor, which helps to get the speed of the vehicle, Arduino microcontroller, and motor controller. The signals from the sensor allows the microcontroller to calculate the velocity and acceleration of the vehicle. According to the velocity and acceleration calculated, the microcontroller modifies the throttle signal that is transferred to the motor controller. In this way, the throttle signal may be regulated and a smooth acceleration is achieved.

\subsection{Sensors}

The first senor used is a Hall Effect sensor. The sensor is mounted close to wheel hub, where part of the axle is none circular. When the concave part is below the sensor, the sensor will return a HIGH signal to the Arduino board, and vice versa. As shown in the graph, in one full rotation, the signal will change from HIGH to LOW for 3 times.

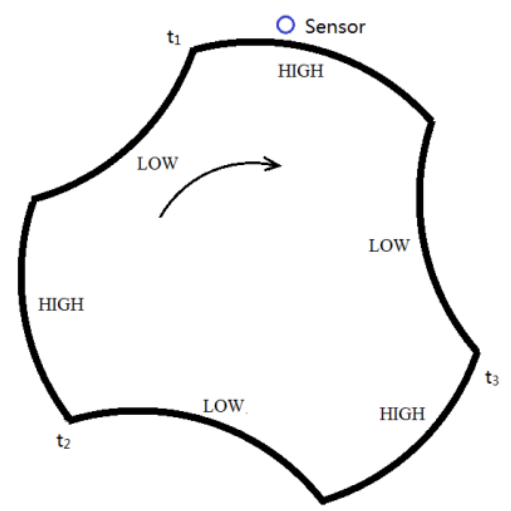

Figure 2: Hall Effect sensor and drive shaft

Suppose $\mathrm{t} 1, \mathrm{t} 2$, and $\mathrm{t} 3$ are time when the sensor value rises in one revolution sequentially. The average speed between $\mathrm{t} 1$ and $\mathrm{t} 2$ can be calculated as following

$$
v_{1}=\frac{r * 2 \pi}{3 *\left(t_{2}-t_{1}\right)}
$$

The average acceleration between $\mathrm{t} 1$ and $\mathrm{t} 3$ is

$$
a_{1}=\frac{2 *\left(v_{2}-v_{1}\right)}{t_{3}-t_{1}}
$$

With a wheel radius of $0.3 \mathrm{~m}$ and a peak speed of $100 \mathrm{~km} / \mathrm{h}$, the time between each signal rise is

$\mathrm{t}=\frac{3.6 * 0.3 * 2 \pi \div 3}{100}=0.0226 \mathrm{~s}=22.6 \mathrm{~ms}$

Thus, the microcontroller need to be able to perform all process within $22.6 \mathrm{~ms}$.

The second sensor used a small generator, whose shaft is synchronised with the motor shaft and rotate at the same rpm as the motor. As the generator rotates, a voltage proportional to the rotational velocity of the shaft is generated. By measuring the voltage generated with the microcontroller, it is possible to derive the velocity of the vehicle.

\subsection{Arduino microcontroller}

The on-board microcontroller is an Arduino Mega 2560 , which has a processing speed of $16 \mathrm{MHz}$. The microcontroller is chosen because of it is 
relatively user friendly and multifunctional. It is able to read both digital and analog signals. The board also provides $3.8 \mathrm{~V}$ and $5 \mathrm{~V}$ output for sensors. Though being a single thread processor, Arduino microcontroller has a designated function, which is named attachInterrupt, that separate sensor monitoring and main programme. [4]

The attachInterrupt function monitors the signal state of the Hall Effect sensor while the main programme runs. When a specific state change takes place, the main programme is paused and a designated function run for once before the main programme resumes. This designated function usually changes some variables which trigger other functions in the main programme.

\section{Throttle control algorithm}

With the hardware in place, we need a control algorithm that would responds quickly to change in motion of the vehicle, as well as be of high reliability that there will be no malfunction.

\subsection{Hall Effect sensor}

As stated above, the signal change in the Hall Effect sensor is at the level of tens of milliseconds. To be able to control the acceleration of the vehicle precisely, it is necessary to measure the speed and acceleration of the vehicle as precise as possible. Because the number of signal change we receive each revolution is relatively low. If we count the number of signal change recorded in a given time frame, the reading is in discreet form as presented in the table below.

\begin{tabular}{|c|c|c|}
\hline $\begin{array}{l}\text { No of valid } \\
\text { signals } \\
\text { recorded }\end{array}$ & $\begin{array}{l}\text { Speed at } \\
50 \mathrm{~ms} \text { interval } \\
(\mathrm{km} / \mathrm{h})\end{array}$ & $\begin{array}{l}\text { Speed at } \\
200 \mathrm{~ms} \text { interval } \\
(\mathrm{km} / \mathrm{h})\end{array}$ \\
\hline 1 & 33.9 & 8.5 \\
\hline 2 & 67.9 & 17.0 \\
\hline 3 & 101.8 & 25.4 \\
\hline 4 & 135.7 & 33.9 \\
\hline 5 & 169.6 & 42.4 \\
\hline
\end{tabular}

Table 1. Precision level at different data logging frequency

Another way to measure the rotation speed of the wheel is to measure the time for the wheel to travel a certain distance. In this case, we record the time when the signal of the sensor change from HIGH to LOW. This can be done using the
attachInterrupt function provided in Arduino microcontroller. There are three variables, $\boldsymbol{t} \mathbf{1}, \boldsymbol{t} \mathbf{2}$, and $\boldsymbol{t} 3$, for time recording in the programme. When the microcontroller detects a signal change from HIGH to LOW, the value of the $\boldsymbol{t} \boldsymbol{l}$ and $\boldsymbol{t} \boldsymbol{2}$ is assigned to $\mathbf{t} \mathbf{2}$ and $\mathbf{t} \mathbf{3}$ respectively. Then the current time is written to $\boldsymbol{t} \boldsymbol{l}$. In this way, the programme always keep record of the time of last three signal changes. And the average velocity between the last two signal changes can be calculated.

$$
v_{1}=\frac{d * \pi}{3 *\left(t_{2}-t_{1}\right)}
$$

Using the first and third variable, the acceleration during the last three signal change can be derived.

$$
a_{1}=\frac{2 *\left(v_{2}-v_{1}\right)}{t_{3}-t_{1}}
$$

Following is the flow chart for the main programme of the Hall Effect sensor.

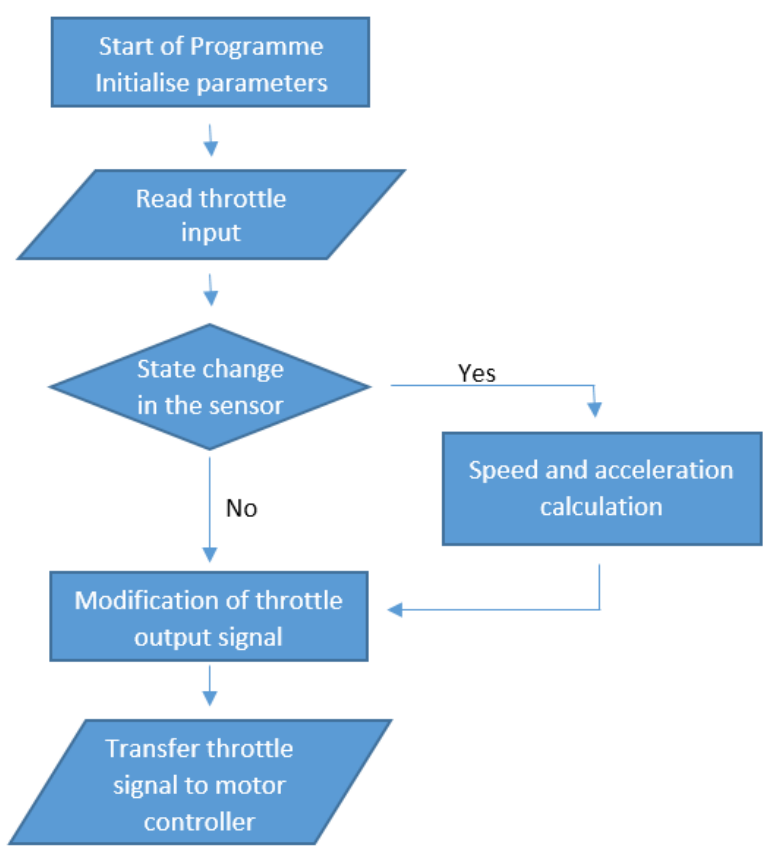

Figure 3: Main programme flow chart

The main programme will always go through the process of throttle signal input, adjusting and output.

\subsection{Regenerative rpm sensor}

The algorithm for regenerative $\mathrm{rpm}$ sensor is relatively straight forward. Velocity value can be 
derived from the voltage of generated by the sensor using the following equation.

$$
v=\frac{V * d * \pi * 60}{r_{g}}
$$

By recording the time when the velocity is measured, the programme can derive the average acceleration for a certain period. Because the microcontroller processes at high speed, the duration between each velocity measurement can be as short as tens of milliseconds. Thus, the acceleration calculated can be assumed to be the acceleration at the instance.

\subsection{Throttle control algorithm}

The parameters for the adjustment includes previous throttle output signal and vehicle acceleration. For throttle control, three different variable related to the control logic are utilised.

\begin{tabular}{|c|l|}
\hline $\begin{array}{c}\text { Sensor value } \\
\text { (T1) }\end{array}$ & $\begin{array}{l}\text { The value measured from } \\
\text { throttle position sensor. This } \\
\text { value may be modified before } \\
\text { used as output value, } \\
\text { depending on other parameters } \\
\text { in the modification logic. }\end{array}$ \\
\hline $\begin{array}{c}\text { Previous output } \\
\text { value (T2) }\end{array}$ & $\begin{array}{l}\text { Previous throttle output value. } \\
\text { Assigned at the end of the } \\
\text { programme. }\end{array}$ \\
\hline $\begin{array}{c}\text { Steady state } \\
\text { value (T3) }\end{array}$ & $\begin{array}{l}\text { When the acceleration is less } \\
\text { than the threshold at, the } \\
\text { previous output value is } \\
\text { assigned to this variable. }\end{array}$ \\
\hline
\end{tabular}

Table 2. Throttle variables used for control algorithm

When the measured acceleration $\mathbf{a}_{\mathbf{m}}$ is greater than the threshold value $\mathbf{a}_{t}$, the throttle output value is modified using the following equation.

$$
\mathrm{T} 1=\mathrm{T} 3+\frac{\mathrm{T} 1-\mathrm{T} 3}{k * \mathrm{a}_{\mathrm{m}}}
$$

By changing the value of at and $\boldsymbol{k}$, the effect of throttle modification can be changed.

Initial test shows that the algorithm is functional in terms of limiting the throttle signal. However, the algorithm does not perform as expected in certain occasions. When the vehicle is accelerating and the throttle position sensor gives a rapidly high throttle signal constantly, T1 may be reduced such that the acceleration drops below the threshold $\mathbf{a}_{\mathrm{t}}$. The steady state value $\mathbf{T 2}$ may be updated to a new value that is much higher than the previous one and increase the acceleration rapidly as well.

To prevent this from happening, a secondary control algorithm is added to the program to ensure that the throttle does not go out of control.

$$
\mathrm{T} 1=\mathrm{T}_{\mathrm{ave}}+\left(\mathrm{T} 1-\mathrm{T}_{\mathrm{ave}}\right) * j
$$

$\mathrm{T}_{\mathrm{ave}}$ is the average value of the latest five throttle output to the motor controller. $\boldsymbol{j}$ is the parameter to adjust the effectiveness of the algorithm. In this way, the growth rate of throttle signal to motor controller is limited regardless of the current acceleration reading.

\section{Experiment}

\subsection{Hall Effect sensor}

Several experiment have been carried out using the Hall Effect sensor. However, strong vibration of the sensor, which results in false measurement, occurs from time to time.

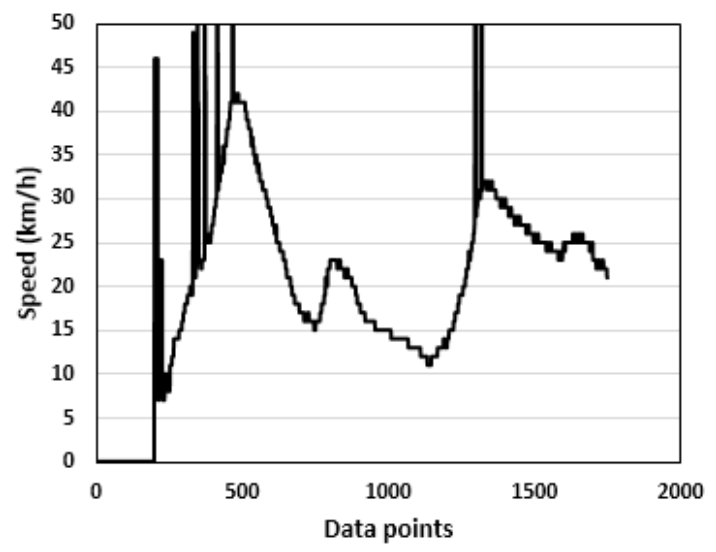

Figure 4: Speed measurement using Hall Effect sensor

\subsection{Regenerative rpm sensor}

Fast and reliable speed measurement is achieved with the regenerative rpm sensor. A few tests are carried to examine the effectiveness of our algorithm. In all these tests, the input throttle signal to the controller is set to maximum value till the vehicle travel a distance of 70 meters. Certain parameters of the two control algorithm are altered to test the effectiveness in different tests.

In the first set of tests, the effectiveness of the feedback algorithm is examined. During the tests, the value of $\boldsymbol{k}$ is changed in different runs, while 
the value of $\boldsymbol{j}$ is kept constant. The test results is show in the graph below.

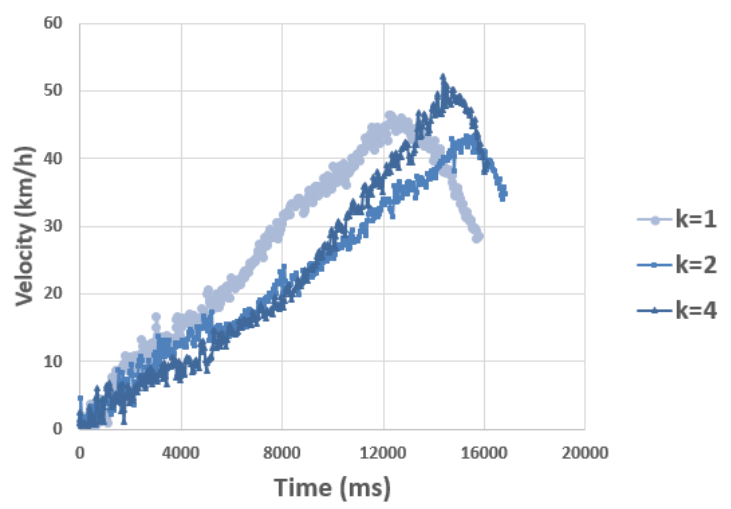

Figure 5. Feedback algorithm test result

As shown in the figure, a large value for $\mathrm{k}$ does not garrenty a slower acceleration. The difference in velocity profile may result from variation in road condition and vibration in the drive train.

The power consumption is also recorded during this set of tests using Cycle Analyst data logger. Following is the power vs. time graph.

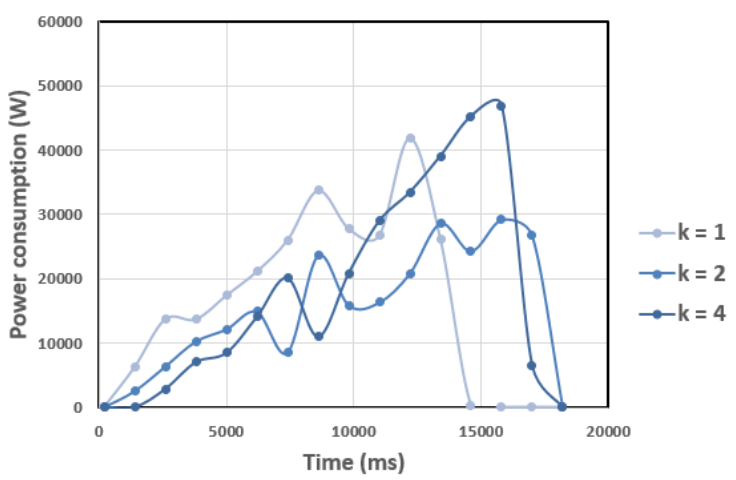

Figure 6. Power vs. time graph

From the graph, we can tell that the power output of the vehicle is lower when the vehicle is going at lower speed and acceleration. Thus, by limiting the acceleration, it is possible to lower the total power consumption during a ride which frequent acceleration and deceleration is present.

In the second set of tests, the value of $\boldsymbol{j}$ is changed to test the effectiveness, while $\boldsymbol{k}$ is constant. Following graph is the velocity vs. time profile of the tests. And the table lists the time spent to travel 70 meters with different $j$ value.

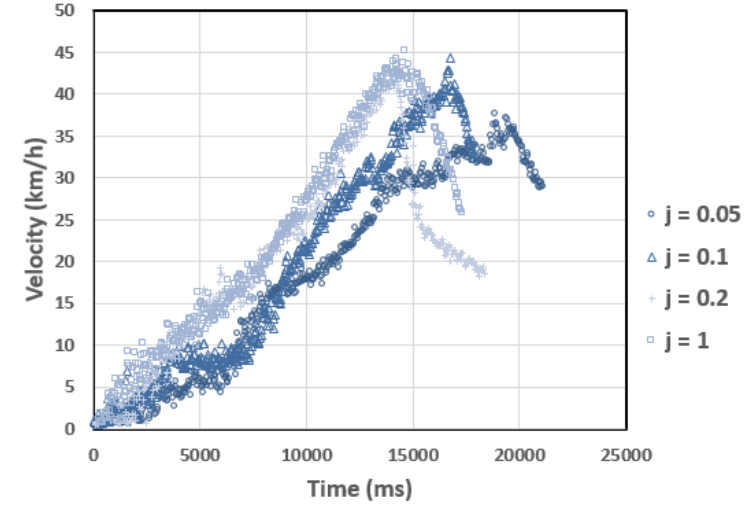

Figure 7. Averaging algorithm test result

\begin{tabular}{|c|c|}
\hline $\boldsymbol{j}$ & Duration(s) \\
\hline 0.05 & 19.5 \\
\hline 0.1 & 16.7 \\
\hline 0.2 & 13.9 \\
\hline 1 & 14.1 \\
\hline
\end{tabular}

Table 3. Time to travel 70 meters

As the result shows, when the value of $\boldsymbol{j}$ is 0.1 or 0.05 , we can observe a significant increase in travel time, which means the acceleration is reduced. For the velocity vs. time profile, it is clear that when the value of $\mathrm{j}$ is smaller, the acceleration is slower and the peak speed is lower at the end of the given distance.

\section{Conclusion}

Test results show that a regenerative rpm sensor is more suitable for feedback control in electric vehicle than a Hall Effect sensor because of its fast and reliable measurement. The experiment also shows that the secondary control algorithm is more effective than the feedback algorithm in terms of giving a smoother start for the vehicle. Though the secondary control algorithm is more effective when the vehicle performs a cold start, it is necessary to have the feedback algorithm because the throttle output increases continuously under the secondary algorithm if the input throttle is increasing despite the acceleration value. Other than this, there is still slight fluctuation in acceleration. This is believed to result from instability of the motor controller when the vehicle is going at low speed. Further improvement and tests will be carried out to optimise the algorithm and improve the acceleration control of the vehicle and further relate it to energy efficiency. 


\section{Acknowledgments}

Sincere thanks to Mr Jiang Zheng, Mr Zu Tianqi, and $\mathrm{Mr}$ Jacob John for their knowledge in Arduino and serial communication.

Sincere thanks to Design Centric Programme for their support to Electric Caterham team.

\section{References}

[1] Davis, T., 2014, THE ELECTRIC CAR

BOUNCES BACK: MILESTONES, Sydney

Morning Herald, Sep 6, 2014. Sydney, N.S.W,

Fairfax Digital, p. 3.

[2] Larminie, J., and J. Lowry, 2003, Electric Vehicle Technology Explained: Chichester, West Sussex, England, J. Wiley.

[3] Hu, J. J., Y. Ji, and R. Du, Control Strategy for Starting and Acceleration of Pure Electric Vehicle.

[4] Loflin, L. Hardware Interrupts Demo-Tutorial for Arduino. Retrived from: http://www.bristolwatch.com/arduino/arduino_ir q.htm

\section{Authors}

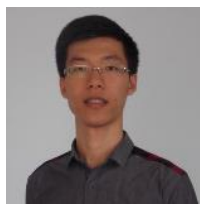

Zhang Kun is an undergraduate student majoring Mechanical Engineering in National University of Singapore. $\mathrm{He}$ has a strong interest in the application of mechatronic system in electric vehicles. He is currently working the improvement of throttle control of the Electric Caterham under Design Centric Programme, Faculty of Engineering.

Joerg Dieter Weigl from Germany (*1977,Ulm), started in his youth with solar vehicles, won in the young research competition. He studied mechanical engineering in Germany and did his Engineering Doctorate on a fuel cell powered motorbike at "Universiti Teknologi Malaysia". He is currently working for National University of Singapore as Lecturer in the Future Transportation Track of the Design Centric Program. He completed several full scale electric vehicle project on land, sea and air. 